\title{
Eigen-Space Coding as a Means to Support Privacy in Computer Mediated Communication
}

\author{
Joëlle Coutaz*, James L. Crowley**, François Bérard* \\ CLIPS-IMAG, BP 53, 38041 Grenoble Cedex 9 \\ (**) INPG, 46 av. Félix Viallet, 38330 Grenoble Cedex \\ FRANCE \\ $\{$ Joelle.Coutaz, jlc, Francois.Berard $\} @$ imag.fr
}

\begin{abstract}
The current solutions to disclosing privacy in computer-mediated communication are two-fold: either the connection is permitted and an audio-video link is opened providing a full-blown perceptual view on the distant location, or the connection is denied and the distant visitor has no perceptual access to the remote site. We claim that the disclosure of private data is more complex than these simplistic binary solutions. In this article. we present the concept of published observability as a system property to characterize the disclosure of private data. We propose a technical solution as an instantiation of this property using an eigen-space coding for private video data. This technique has been implemented within CoMedi, a mediaspace under development.
\end{abstract}

KEY WORDS privacy, computer mediated communication, mediaspace, eigen-space coding, computer vision

\section{INTRODUCTION}

Computer Mediated Communication (or CMC) covers multiple forms of person-to-person communication supported by a computer network infrastructure. CMC may occur asynchronously as with E-mail or synchronously as in desk-top video conferencing and mediaspaces. In typical mediaspace settings such as Cavecat (Mantei, 1991), Cruiser (Fish, 1992) and Montage (Tang, 1994), users can teleglance at a remote office, open a Vphone connection or maintain a permanent link with a distant shared location. Although these systems are intended to support informal encounterings and provide a global sense of a shared community, they may also be used abusively and highjack our own privacy.

Access control to privacy may rely on social protocols as in the very first mediaspace developed at PARC (Stults, 1986); it may rely on technical solutions as in Cruiser, or it may include a combination of both imperative and indicative controls as in Montage. The current solutions to disclosing privacy are two-fold: either the connection is permitted and an audio-video link is opened providing a fullblown perceptual view on the distant location, or the connection is denied and, with the exception of Cruiser, the distant visitor has no perceptual access to the remote site. We consider that the disclosure of private data is more complex than these simplistic binary solutions.

In this article, we present the concept of published observability as a system property that characterizes the disclosure of private data to distant users. We propose a technical solution as an instantiation of this property using an eigen-space coding for private video data. This technique has been implemented within CoMedi, a mediaspace under development. 


\section{COMEDI, A MEDIASPACE PROTOTYPE}

\footnotetext{
CoMedi (Communication and Media-space) is being rested on four Silicon Graphics Indy workstations communicating over the Ethernet. Each workstation is equipped with a motorized pan-tiltzoom color camera. and microphones placed on the four corners of the computer monitor. It provides users with typical facilities such as glancing at someone, Vphoning and exploring a remote location using a virtual window (Gaver, 1995): the distant camera is remotely controlled by the user's head movement.

In its current state, the originality of CoMedi lies in the integration of multiple active and cooperative computer vision processes: three visual processes (eye blink detection, skin-driven color histogram (Schiele, 1995), and cross-correlation (Crowley, 1995)) are used in cooperation to detect and track mediaspace occupants with robust and real time performance (Coutaz, 1996). The availability of complementary visual processes permits the system to interpret the user's movements by activating the set of processes that are appropriate to the situation. In particular, users can accomplish their tasks without distractions of remaining in a narrow field of view. They can move around and adjust their own views on remote sites without being tied up to the workstation.

The cooperation of multiple visual processes is primarily intended for improving the robustness and the flexibility of the tracking system. It also serves as the foundation for increasing the transmission bandwidth. Low bandwidth is an important source of discontinuities within the human communication process (Sellen, 1995). It can be improved by using high speed ATM based-technology, complemented with compression techniques. In CoMedi, we obtain very high video compression ratio by stabilising the image of the user using our visual cooperating processes. Stabilisation provides the position. scale and illumination normalisation which makes possible efficient compression using an eigen-space. In CoMedi, eigen-space coding was initially intended for increasing transmission bandwidth. Early experiments led us to exploit this technique for an additional
}

purpose: that of a magic filter for published private video data.

\section{THE CONCEPT OF PUBLISHED OBSERVABILITY}

A number of properties have been developed in the literature to structure and reason about the design, the implementation and the evaluation of interactive systems (IFIP WG2.7, 1996; Dix, 1991). One of these properties is "Observability": the property that the presentation of the system contains sufficient information to allow the user to determine the functional state of the system (Salber, 1994; Salber 1995). In principle, the information to be made observable is motivated by the task model.

In groupware, observability may be in conflict with the protection of the private space. The private space is the set of variables that model personal data, for example, the fact that I am currently in my office reading my E-mail. The quality of being "personal" for a particular state variable is decided at the analysis stage of the design process. Clearly, the observability of personal state variables is relevant to group awareness (Dourish, 1992) but may conflict with privacy. Our concept of published observability allows designers to reason about this antagonism (Salber. 1995).

A personal state variable relevant to a group activity is made observable only if its owner has authorized its publication. The authorization for publication may be static (i.e., decided at the design stage) or dynamic (i.e., may be changed at run time). For ethical reasons, the owner of a state variable should be the user whose state is conveyed by the variable but the owner may lend the state variable to others.

The binary duality "authorization-interdiction" of publishing a state variable does not convey the subtlety of human social relations. We introduce the concept of publication filter to support this requirement. A filter is both a mapping and a transformation function. As shown in Figure 1, a publishable variable $P$ is linked to at least one representative variable $\mathrm{R}$. In turn, $\mathrm{R}$ is rendered by at least one presentation object that makes $\mathrm{R}$ perceivable 
to remote observers. Dependencies between $\mathrm{P}$ and $\mathrm{R}$ and between the representatives and the presentation objects are maintained by the publication filters. The type of filter depends on $\mathrm{P}$, on the role of the owner. on social relationships. etc. The identity function is one such basic filter. The eigen-space coding described in Section 4, provides an innovative filter for private video data.

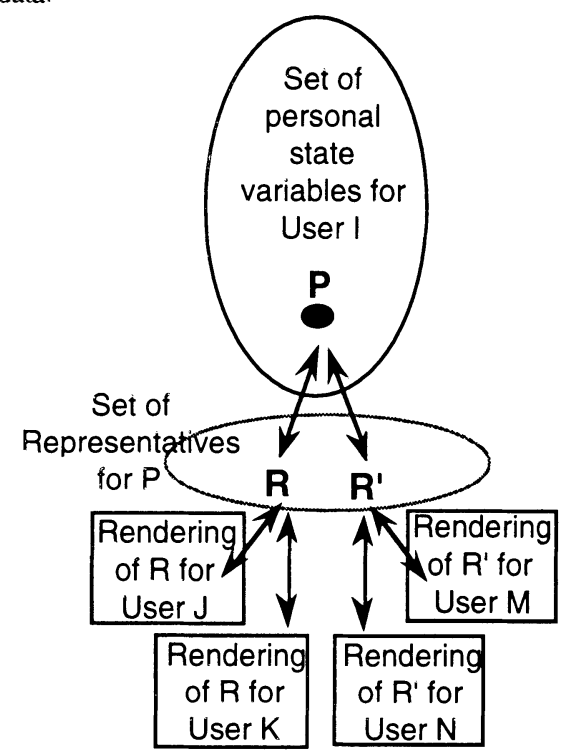

Figure 1. Published and filtered observability. Arrows denote publication filters. In this illustration, personal state variable $P$ is published as two representatives $R$ and $R$ ' for two different groups of users. In turn, $R$ and $\mathrm{R}^{\prime}$ are rendered depending on the final observer in each group.

For example, "current activity" can be defined as a personal state variable $P$ that memorizes the current activity (e.g., reading E-mail) of a user engaged in a mediaspace. The user may decide to publish this variable as is for his collegues (therefore, the filter is the identity function) but, for unknown users, he may decide to hide himself behind a smoke screen. For example, one possible representative $\mathrm{R}$ for $\mathrm{P}$ would be the level of availability. $R$ would then be rendered by an interaction object (e.g., an icon) using the door metaphor as in Cavecat, or the venitian blinds metaphor as in Cruiser, or even the shadow filter as proposed in (Hudson, 1996).

In this section, we have introduced the concepts of published observability and publication filter. We have illustrated these notions with known solutions as developed for current mediaspaces. In the next section, we describe a new way of handling video images using our concept of published and filtered observability.

\section{EIGEN-SPACE CODING AS A PUBLICATION FILTER}

Eigen-space coding employs principal components analysis to determine an optimal basis space for coding a signal for transmission(Loève, 1963; Watanabe, 1965). This mathematical tool has been used recently in computer vision for recognition: identifying faces (Turk, 1991), interpreting hand gesture (Martin, 1995), and indexing documents. As discussed above, we have used eigen-space coding as a compression technique for video-phone images as well as for the basis of a publication filter. The computational details of the technique is available in the Annexe as well as in (Brégeon, 1996). In this section, we only outline the principles.

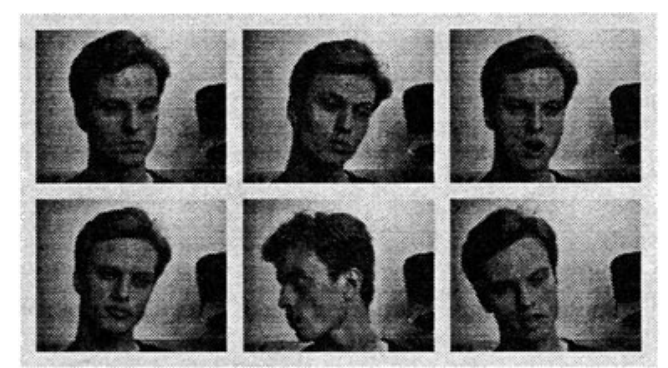

Figure 2. Sample images $I_{0}, I_{1}, \ldots I_{5}$ used to build an eigen-space for Laurent.

Video images that are sent over the network through a publication filter are coded as their coordinates in an $\mathrm{N}$-dimensional orthogonal space: an eigen-space. This space is defined as the principal components computed from a set of representative 
video images. Figure 2 shows a sample of 6 initial images $I_{0}, I_{1}, \ldots I_{5}$ used to build the eigen-space $J_{0}$, $J_{1}, \ldots . J_{4}$ shown in Figure 4. Figure 3 shows the mean image of the sample images. The received image is rebuilt in real time on distant sites using the mean image and a linear combination of the basis images.

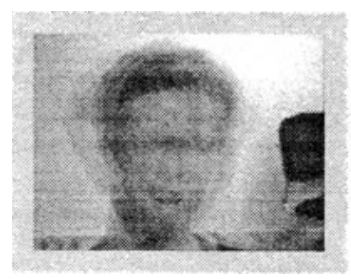

Figure 3. The mean image $T=\frac{1}{n} \sum_{k=0}^{n-1} I_{k}$
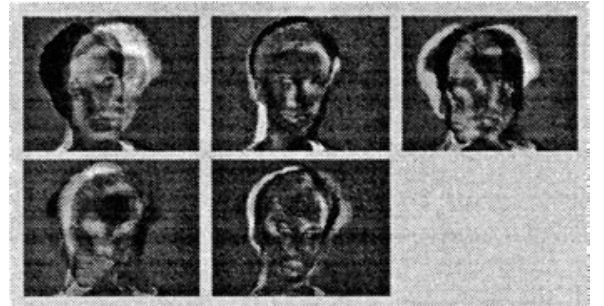

Figure 4. The resulting eigen-space $J_{0}, J_{1}, \ldots . J_{4}$ for sample images of Figure 2.

Figure 5 shows a live source image of Laurent and its reconstruction as perceived by a distant observer. It illustrates how the publication filter works. We observe that the reconstruction process has filtered out Laurent's arm: his representative published set, $I_{0}, I_{1}$, $\ldots \mathrm{I}_{5}$, does not include any image with an arm raised. As a result, any person appearing in the background, Laurent picking his nose, or even Laurent being naked would not be published to distant observers. Laurent could even use a John Wayne eigen-space to reconstruct his live images on the fly. (We have played this trick using another student's eigen-space to reconstruct Laurent's live faces.)

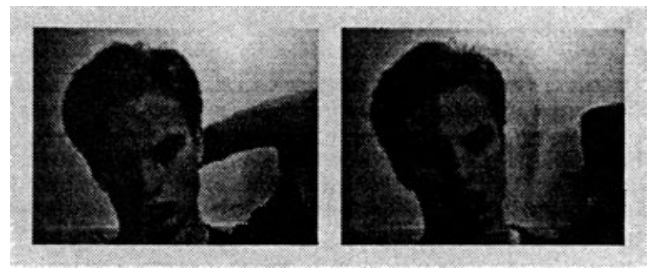

5a) $W=$ Source live image 5b) $\widetilde{W}=$ Received rebuilt image

Figure 5. A source live image $W$ of Laurent (5a) and its reconstruction $\tilde{W}$ as perceived by a distant observer (5b) $\left.W=T+\sum_{i=0}^{i=p-1} d i\right) \cdot J$ where $c(i)$ denote the coordinates of $W$ in the eigen-space $J_{b b} J_{1}, \ldots . J_{4}$ of figure 2 .

The reconstruction is perfect when the live image is identical to one of the sample images. When not identical but reasonably similar, the reconstructed image is slightly distorted (see Figure 6). Real life requires more precision. To support this new requirement, we have developed an incremental eigenspace coding capable of adjusting dynamically to local changes: when the energy of the current live image exceeds a threshold, the local as well as the distant eigen-spaces for that user are updated accordingly (Brégeon, 1996).

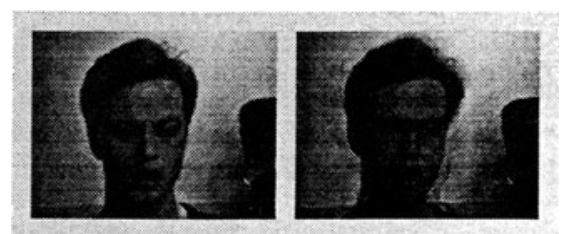

Figure 6. Live source image (left) and its real time reconstruction (right) when the source image is within a reasonable distance in the eigen-space. 


\section{CONCLUSION}

The concept of published observability and its companion, the notion of publication filter, provide a new dimension for reasoning about privacy in multiuser systems. We have exploited a new way of using eigen-space coding for publishing private video data in an innovative and flexible manner. So far, we have demonstrated the technical feasibility of our ideas through a real time implementation of the eigen-space filter on workstations linked over Internet. Clearly, ATM-based technology would significantly improve the incremental version of the eigen-space filter.

We have not yet performed usability studies. We need to evaluate the impact of image distorsion in relation to communication tasks as well as the social implication of hiding or transforming "real pixels". In the current implementation of CoMedi, we do support reflexivity (the user sees his reconstructed image) in order for the sender to check the image received on remote sites.

We also claim that one filter does not fit all. Filters should be composed over areas of the live scene to provide a flexible and fine grain level of privacy for video data. For example, I would like the system to replace the area of the blackboard in my office that discloses my latest scientific results with a nice poster image. I would also like to provide the remote observer with a sense of coming and going activities at my office door using a shadow filter (Hudson. 1996). In addition. I would like to use the eigen-space filter for my face so that no one sees when I am sucking my pen while typing at the terminal. To this end, we have developed a robust face tracker (Coutaz, 1996, Crowley, 1997) that controls a pan-tilt-zoom camera and provides the eigen-space filter with a normalized source image.

Composing video filters with sound information is another interesting avenue for novelty and questions: How eigen-space coding can be extended to speech and sound to provide remote users with sufficient awareness of local sound events without having access to the real wording? More generally, how should filter composition be made amenable to non specialist users?

\section{ACKNOWLEDGEMENT}

This work is supported by France Telecom-CNET. We are grateful to Denis Brégeon and Laurent Le Corre for the implementation of the eigen-space coding algorithms.

\section{ANNEX}

\section{Eigen-Space Coding}

\section{Notations}

Upper cases denote vectors and matrices. Lower cases correspond to scalar values.

A source image whose definition is $\mathrm{w} x \mathrm{~h}$ pixels is represented by a vector composed of $w^{*} h$ components. Each component represents one pixel of the source image.

The number of images in the initial sample set is n.

The number of dimensions retained for the EigenSpace is $\mathrm{p}$.

Initial set of sampled images: $I_{k} \quad \forall \mathrm{k}=0,1, \ldots$ n-1

A mean image is computed from the initial sampled set: $\quad T=\frac{1}{n} \sum_{k=0}^{n-1} I_{k}$

The sampled images are recentered on the mean image: $T_{k}=I_{k}-T \quad \forall \mathrm{k}=0,1, \ldots \mathrm{n}-1$.

Matrix of recentered images: $\mathrm{A}=$ $\left[\begin{array}{llll}T_{0} & T_{1} & \ldots & T_{n-1}\end{array}\right]$ of A)

Covariance Matrix: $\mathrm{A} \mathrm{A}^{\mathrm{t}}\left(\mathrm{A}^{\mathrm{t}}\right.$ denotes the transpose

Working matrix: $A^{t} A=Q$

Eigen vectors of the working matrix: $\quad V_{k} \forall \mathrm{k}$ $=0,1, \ldots \mathrm{n}-1$ (ranked according to decreasing eigenvalue)

Eigen vectors of the sampled set: $Z_{k}=\sum_{i=0}^{i=n-1} V_{k}(l) \cdot T_{i} \forall \mathrm{k}=0,1, \ldots \mathrm{p}-1$ 
Eigen-space is composed of the normalized eigen vectors: $J_{k}=\frac{Z_{k}}{\| Z_{k} \mid} \quad \forall \mathrm{k}=0,1, \ldots \mathrm{p}-1$

\section{Algorithm}

The construction of the eigen-space follows the following steps:

1. Sample $\mathrm{n}$ initial images representing publishable scenes (see Figure 2): $I_{0}, I_{l}, \ldots I_{n-1}$

2. Compute the mean image $\Gamma$ of the sampled set (see Figure 3 ) as well as the set of initial images recentered on the mean image, e.g., for every $i$ compute $T_{i}$ and build the matrix $\mathrm{A}=$ $\left[\begin{array}{llll}T_{0} & T_{1} & \ldots & T_{n-1}\end{array}\right]$

Reduce A to a set of orthogonal images using principal component analysis (see figure 4 ). The orthogonal images are defined as the eigen vectors of $A A^{t}$. However $\mathrm{A} \mathrm{A}^{t}$ has a size which is the square of the number of pixels in each image. In most cases it is unreasonable to compute such a matrix. To avoid this problem we consider the matrix of inner products between the images, $\mathrm{Q}=$ $A^{\prime} A$. We note that the Eigen-vectors of $Q$ are $\mathrm{Q} \mathrm{R}=\lambda \mathrm{R}$. We note the following identity:

$$
\begin{aligned}
& \left(\mathrm{A}^{\prime} \mathrm{A}\right) \mathrm{R}=\lambda \mathrm{R} \\
& \mathrm{A}\left(\mathrm{A}^{\prime} \mathrm{A}\right) \mathrm{R}=\mathrm{A} \lambda \mathrm{R} \\
& \left(\mathrm{AA}^{t}\right) \mathrm{AR}=\lambda \mathrm{AR}
\end{aligned}
$$

Thus $\lambda$ AR give the eigen-vectors of $\mathrm{AA}^{\prime}$.

3. Order $V$ according to their decreasing eigen-value

so as to select the $p$ vectors among $n$ that are the most discriminants. Chose $\mathrm{p}$ so that the sum of the eigenvalues attains a threshold.

The eigen-space of publishable scenes is used locally to code source images in real time as a vector of $p$ numbers. This vector is transfered to the remote site where the image is reconstructed using the eigenspace sent once at connexion time.

Source live image : W

$$
\begin{aligned}
& \text { Projection vector: } \quad \mathrm{C}=\{\mathrm{c}(\mathrm{i})\} \quad \text { with } \mathrm{c}(\mathrm{i}) \\
= & <\mathrm{W}-T, \mathrm{~J}_{\mathrm{i}}>, \mathrm{i}=0, \ldots \mathrm{p}-1
\end{aligned}
$$

$\mathrm{C}$ is a column vector built from the projection of $\mathrm{W}$ recentered with the mean image.

Rebuilt images are computed as:

$$
W=T+\sum_{i=0}^{i=p-1} d(i) \cdot J
$$

\section{REFERENCES}

Brégeon, D. and Le Corre L. (1996) Technique de Codage par Eigen Vecteurs, Rapport de projet de fin d'étude ENSIMAG, 1996.

Coutaz, J. , Crowley, J. L. and Bérard, F. (1996) Coordination of Perceptual Processes for Computer Mediated Communication, in proceedings of the second International Conference on Automatic Face and Gesture Recognition, IEEE Computer Society Press, pp. 106-111.

Crowley, J. L. and Martin. J. (1995) Experimental Comparison of Correlation Techniques", in proc. IAS-4, International Conference on Intelligent Autonomous Systems, Karlsruhe.

Crowley, J. L. and F. Bérard (1997) Multi-Modal Tracking of Faces for Video Communications, IEEE Conference on Computer Vision and Pattern Recognition CVPR '97, Puerto Rico.

Dix, A. (1991) Formal Methods for Interactive Systems, Academic Press.

Dourish, P. and Bly, S.A. (1992) Portholes : Supporting Awareness in a Distributed Work Group, in proceedings of the CHI'92 Conference on Human Factors in Computing Systems, pp. 541-547.

Fish, R.S., Kraut, R.E., Root, R.W., and Rice R.E. (1992) Evaluating Video as a Technology for Informal Communications, in proceedings of the CHI'92 Conference on Human Factors in Computing Systems, pp. 37-47.

Gaver, W., Smets, G. and K. Overbeeke (1995) A Virtual Window on a Media Space, Proc. CHI'95, ACM publ., pp. 257-264.

Hudson, S. \& Smith, I. (1996) Techniques for Addressing Fundamental Privacy and Disruption 
Tradeoffs in Awareness Support Systems. In Proc. CSCW'96, ACM Press, pp. 248-257.

IFIP WG 2.7 (1996) Design Principles for Interactive Software (C. Gram \& G. Cockton Eds.), Chapman\&Hall.

Loève, M. (1963) Probability Theory, 3rd Edition, Princeton, Van Nostrand.

Mantei, M., Backer, R.M., Sellen, A., Buxton, W., Milligan, T. and Wellman B. (1991) Experiences in the use of a Media Space, in proceedings of the CHI'91 Conference on Human Factors in Computing Systems, pp. 203-208.

Martin, J. (1995) Suivi et Interprétation de Geste : Application de la Vision par Ordinateur à l'Interaction Homme-Machine, Rapport DEA Informatique, IMAG - INPG.

Salber, D. (1994) The System Modelling Glossary, Amodeus Project Document: System Modelling/WP 26.

Salber, D. (1995) De l'interaction Homme-Machine individuelle aux systemes multi-utilisateurs: l'exemple de la communication homme-homme médiatisée, Thèse de doctorat de l'Université Joseph
Fourier, Grenoble.

Schiele, B. and Waibel, A. (1995) Gaze Tracking Based on Face Color, in proc. International Workshop on Face and Gesture Recognition, Zurich. Sellen, A. (1995) Remote Conversations: The Effects of Mediating Talk with Technology, in Human Computer Interaction, Lawrence Erlbaum Publ., Vol. 10(4), pp. 401-444.

Stults, R. (1986) MediaSpace, rapport technique Xerox PARC.

Tang, J.C. and Rua, M. (1994) Montage: Providing Teleproximity for Distributed Groups, in Proc. of the Conference on Computer Human Interaction (CHI'94), pp. 37-43.

Turk, M. and Pentland, A. (1991) Eigenspaces for Recognition. Journal of Cognitive Neuroscience, 3(1), pp. 71-86.

Watanabe, S. (1965) Karhunen-Loève Expansion and Factor Analysis: Theoretical Remarks and Applications, Transaction of the Fourth Prague Conference on Information Theory, Statistical Decision Functions and Random Processes, 1965. 